\title{
Serie temporal de los accidentes laborales atendidos en un hospital de agudos y su relación con la meteorología y la polución atmosférica
}

\section{Time series of industrial accidents attended in an acute hospital care and its relation to meteorology and atmospheric pollution}

\section{Brígido Pérez Bermúdez}

Médico del Trabajo del S . Prevención de Riesgos Laborales. Área 2. H. U. de la Princesa de Madrid. España

\section{Antonio Valdivia Pérez}

Médico Residente del S ${ }^{\circ}$. de Medicina Preventiva del H. U. de la Princesa de Madrid.España

\section{Ana Belén Nieto Sánchez}

Enfermera del Trabajo del So. Prevención de Riesgos Laborales. Área 2. H. U. de la Princesa de Madrid. España

\section{Correspondencia}

Dr. Brígido Pérez Bermúdez

Medico del Trabajo

Servicio de Prevención de Riesgos Laborales. Área 2.

Hospital Universitario de la Princesa.

C/ Diego de León 62. 28006 Madrid. España.

Tf: 915202596

bperezb.hlpr@salud.madrid.org

\section{Resumen}

Introducción: La siniestralidad es un proceso en muchas ocasiones complejo en el que entran diversos factores de riesgo, tanto inherentes al trabajo como a circunstancias exteriores (organización, climatología, etc.).

Objetivos: Describir la tendencia y factores relacionados de los accidentes de trabajo (A.T.) atendidos en un hospital de agudos de Madrid durante el trienio 2005-07 y su relación con la meteorología y la polución atmosférica.

Metodología: Estudio ecológico. Se analizaron los A.T. atendidos en el Servicio de Urgencias del Hospital Universitario de la Princesa de Madrid, durante el trienio 2005-07. Las variables analizadas fueron variables de calendario, climáticas y niveles de contaminación. Se realizó un estudio descriptivo y univariante y se modelizó por medio de una Regresión de Poisson. La medida de asociación fue el Riesgo Relativo con sus Intervalos de Confianza al $95 \%$.

Resultados: La tendencia es estacionaria disminuyendo en el año 2007. El calor es un factor de riesgo, temperaturas superiores a $25^{\circ} \mathrm{C}$. aumenta la siniestralidad un $19 \%$. La presión atmosférica se comporta como efecto protector. Las variables de contaminación no fueron significativas.

Conclusiones: Durante el año 2007, la siniestralidad laboral atendida en el servicio de Urgencias del hospital ha disminuido. Los A.T. tienen una relación con temperaturas elevadas y presión atmosférica.

Palabras claves: Análisis serie temporal, accidentes laborales, meteorología y contaminación, Med Segur Trab 2009; 55 (214): 64-71 


\section{Abstract}

|||||||||||||||||||||||||||||||||||||||||||||||||||||||||||||||||||||||||||||||||||||||||||||||||||||||||||||||||||||||||||||||||||||||||||||||||||||||||||||||||||||||||||||||||||||||||||||||||||||||||||||||

Introduccion: The work accident rate is in several occasions a complex process in wich divers safety factors take place, inherente to the job /profesión as well as external circumstances (organization, climatology, etc)

Objectives: To describe the tendency and factors wich are linked to the occupational Hazard, admitted toa cute hospital in Madrid during the 2005-2007 triennium and its relations with meteorology and environmental pollution.

Methodology: Ecologic study. Occupational injuries admitted in the accident and emergency department at the University Hospital "La Princesa" in Madrid were analysed Turing the 2005-2007 triennium. The variables analysed were calendar variable, climatic and levels of environmental pollution. A descrptive and univariant study was carried out under the assumption of a Poisson regresión. The measure of association was relative Risk with its Confidence Interval Applet at 95\%.

Results: The tendency is stabillized but weaning in 2007 . Heat is a risk factor, temperaturas above 25 Degress Celsius increases the work accident rate by $19 \%$. Atmospheric pressure behaves as a protective effect. However, the variables for contamination weren't significant.

Conclusion: During 2007, work accident rate atended in the accident and emergency department at the Hospital has diminish. Occupational Hazard is related to high temperaturas and atmospheric pressure.

Key Words: Time series analysis, Occupational Hazard, meteorology and environmental pollution. Med Segur Trab 2009; 55 (214): 64-71 


\section{INTRODUCCIÓN}

Los accidentes de trabajo (AT) son unas de las consecuencias más temidas en el mundo laboral. España ocupa, en la Comunidad Europea tras Portugal, el segundo puesto en tasas de siniestralidad laboral. Estas cifras en el último año (Julio 2007 a Junio del 2008), que recogen el Instituto Nacional de Seguridad e Higiene de Trabajo es de 919.307 A.T. totales sobre una población afiliada a la seguridad social (S.S.) de 16.102.746. En concreto en Madrid se produjeron 132.492 A.T. sobre una población afiliada a la S.S. de 2.628.662 lo que supone una incidencia de 5.040 A.T por 100000 trabajadores al año.

La siniestralidad es un proceso en muchas ocasiones complejo en el que entran diversos factores de riesgo, tanto inherentes al trabajo, como a circunstancias exteriores (organización, climatología, etc.).

Por otra parte, en el año 1994, Naciones Unidas crea el Convenio Marco de Naciones Unidas del Cambio Climático y en 1997 se publican los acuerdos del protocolo de Kioto, a partir de estos reconocimientos internacionales existe una mayor preocupación por las causas y consecuencias que las alteraciones en el clima puedan tener para los seres vivos. Desde entonces se ha incrementado el número de estudios cuyo objetivo es analizar la relación entre la salud, el clima y la contaminación. En España el Ministerio de Medio Ambiente viene estudiando dicha problemática así como propuestas de adaptación a este fenómeno ${ }^{1,2}$.

El estudio EMECAM ${ }^{3}$ en el año 1999 es el primer estudio multicéntrico que se realiza en España con el fin de estimar los efectos de la contaminación y la mortalidad en las ciudades más importantes del Estado Español.

En la bibliografía consultada son escasos los trabajos que analizan la contaminación y el clima en relación con los A.T. El objetivo de este estudio es describir la tendencia y factores relacionados de los A.T. que fueron atendidos en un hospital de agudos de Madrid durante los años 2005, 2006 y 2007 y su relación con la meteorología y la polución atmosférica.

\section{METODOLOGÍA}

Estudio ecológico. Se analizaron todos los AT que fueron atendidos en el Servicio de Urgencias del Hospital de la Princesa de Madrid, durante los años 2005, 2006 y 2007. En la serie temporal la unidad de registro es el día.

Los datos de actividad asistencial nos fueron suministrados por la Unidad de Información Clínico-Asistencial de este Hospital. Los valores de contaminación ambiental proceden del Área de Medio Ambiente del Ayuntamiento de Madrid, y corresponden a los promedios diarios de 24 estaciones medidoras ubicadas en diferentes puntos de la ciudad. Las variables climáticas se obtuvieron de la Agencia Estatal de Meteorología, y corresponden a la estación de Getafe (3200), a excepción de la radiación Ultravioleta (UV), recogida en la estación de Ciudad Universitaria (3194).

La variable a estudio fue el número de accidentes laborales que fueron atendidas en el día por el Servicio de Urgencias del Hospital. U. de la Princesa y las variables explicativas fueron variables de calendario (día de la semana, mes y días festivos), climáticas (Lluvia, nieve, niebla, viento fuerte, tormenta, radiación ultravioleta, presión atmosférica media al día, días con temperaturas superiores a $25^{\circ} \mathrm{C}$. y días con temperaturas inferiores a $0^{\circ} \mathrm{C}$.) y niveles de contaminantes atmosféricos ( $\mathrm{SO} 2, \mathrm{CO}, \mathrm{NO} 2, \mathrm{O} 3$ y $\mathrm{PM}$ 10). Las variables climáticas se recogieron como dicotómicas y cuantitativas según el caso. Las contaminantes, como continúas con sus valores máximos en el día.

Se realizó un análisis descriptivo y se representó por medio de gráficos de cajas las variables calendario. Posteriormente se calculó la tendencia de los accidentes a partir de suavizado lowess y posterior ajuste a una función polinomial de orden 2 . 
Para el análisis univariado se utilizó el test de Mann-Whitney para variables explicativas con 2 categorías, y correlación de Spearman para continuas.

Por último, se realizó un modelo de regresión de Poisson, siguiendo la metodología propuesta por Sáez M, Pérez-Hoyos S, Tobías A, et $\mathrm{al}^{4}$, en el que se incluyeron las variables de calendario (mes, semana y festivos), climáticas y de contaminación, así como la tendencia. Se eliminaron secuencialmente las variables menos significativas, comprobando que no se producían pérdidas importantes mediante el test de razón de verosimilitud. La medida de asociación es el riesgo relativo (RR) con sus intervalos de confianza (I.C) al $95 \%$.

Posteriormente se generaron las predicciones según el modelo, su límite de confianza superior y realizamos la postestimación con el análisis de autocorrelación de los residuos de Pearson, repitiendo la modelización con términos autoregresivos y comprobación final de ajuste.

Los cálculos y gráficos se realizaron con los programas Microsoft Excel e Intercooled Stata 9.1 para Windows.

\section{RESULTADOS}

Se atendieron durante los 3 años de estudio (2005 al 2007), un total de 911 accidentes laborales, con una media de 2.08 accidentes por día (mediana de 2) y una desviación típica de 1.63. La edad media de los accidentados fue de 36.8 (desv. típica 11.5) años. $70.2 \%$ fueron hombres y 29.8 \% mujeres. Los gráficos de cajas (1 y 2) muestran la distribución de los accidentes durante los días de la semana y durante los meses del año.

Gráfico 1. Diagrama de cajas de los Accidentes Laborales por meses

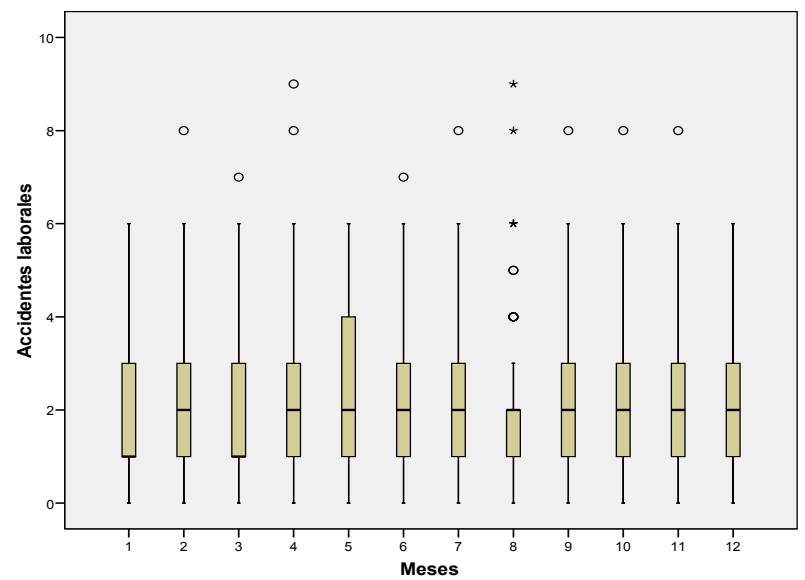

Gráfico 2. Diagrama de cajas de los Accidentes laborales por día de la semana

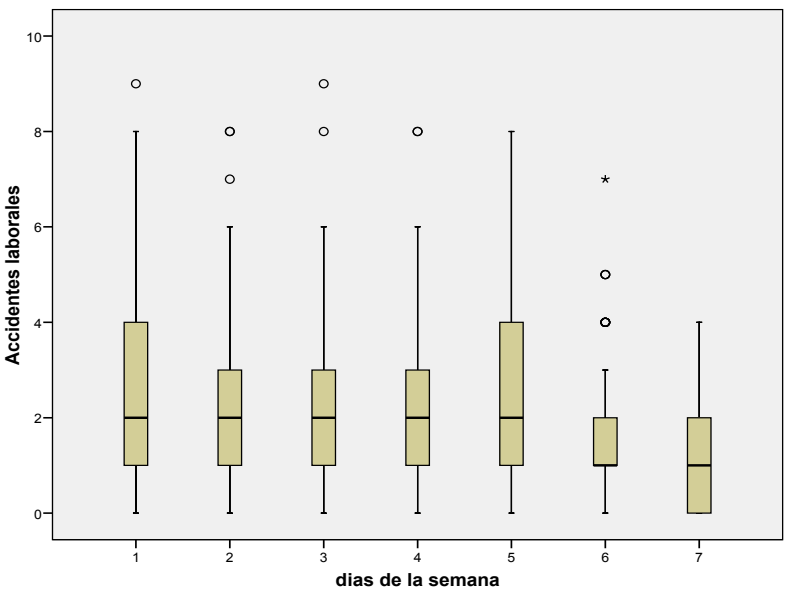

Serie temporal de los accidentes laborales atendidos en un hospital de agudos y su relacion con la meteorologia y la polución atmosferica Brígido Pérez Bermúdez, Antonio Valdivia Pérez, Ana Belén Nieto Sánchez 
Se observa que durante el mes de Agosto existen menos accidentes laborales atendidos.

Respecto al comportamiento durante la semana, los fines de semana y festivos, se atendieron menos accidentes como era de esperar.

La tendencia de esta distribución se refleja en el siguiente gráfico:

\section{Gráfico 3. Tendencia de los accidentes laborales durante el periodo de estudio}

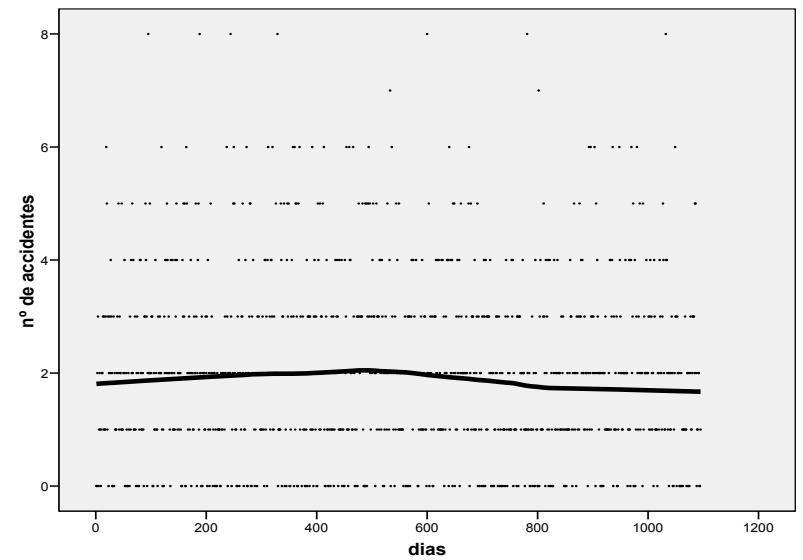

Como se contempla en el gráfico de tendencia, la frecuencia de accidentes laborales atendidos durante el periodo analizado es muy constante, si bien existe un suave descenso a partir de la mitad del periodo del estudio.

La tabla I, muestra el análisis univariante de las variables Meteorológicas y las variables contaminates en relación con los accidentes laborales atendidos en el Servicio de Urgencias del Hospital.

Tabla I. Estudio univariante

\begin{tabular}{|c|c|c|c|}
\hline Variables Meteorológicas & $\mathbf{P}$ & Variables de Contaminación & $\mathbf{P}$ \\
\hline Lluvia * & 0.647 & $\mathrm{SO} 2(\mu \mathrm{g} / \mathrm{m} 3)^{* * *}$ & 0.542 \\
\hline Nieve* & 0.151 & $\mathrm{CO}(\mathrm{mg} / \mathrm{m} 3)^{\text {*** }}$ & 0.588 \\
\hline Tormenta* & 0.597 & $\mathrm{NO} 2(\mu \mathrm{g} / \mathrm{m} 3)^{* * *}$ & 0.001 \\
\hline Niebla* & 0.023 & PM10 $(\mu \mathrm{g} / \mathrm{m} 3)^{* * *}$ & 0.003 \\
\hline Viento fuerte* & 0.398 & $\mathrm{O} 3(\mu \mathrm{g} / \mathrm{m} 3)^{* * *}$ & 0.180 \\
\hline $\mathrm{T}^{\mathrm{o}}$ bajo $0^{\circ} \mathrm{C}^{*}$ & 0.620 & Benceno $(\mu \mathrm{g} / \mathrm{m} 3)^{* * *}$ & 0.061 \\
\hline $\mathrm{T}^{\mathrm{o}}$ superior a $25^{\circ} \mathrm{C} . *$ & 0.031 & & \\
\hline Presión Atmosf. media al día** & 0.046 & & \\
\hline
\end{tabular}

Como puede apreciarse existen diferencias significativas entre los accidentes laborales atendidos con los días de niebla, con temperaturas superiores a $25^{\circ} \mathrm{C}$, con días de cambios de presión atmosférica, con las concentraciones de NO2 y de PM10.

Se realiza un modelo de regresión de Poisson incluyendo todas las variables con $\mathrm{p}<0,2$ en el análisis univariado, así como las variables semana, mes y festivos. Se han eliminado secuencialmente las menos significativas para el modelo (test de razón de verosimilitud $>0,1$, obteniendo los resultados expuestos en la tabla II. 
Tabla II. Resultado final de la Regresión de Poisson

\begin{tabular}{|c|c|c|c|}
\hline Variable & $\mathbf{R R}$ & $\mathbf{P}$ & 95\% Conf. Interval \\
\hline Año 2005 & 1 & & \\
\hline Año 2006 & 0.996 & 0.940 & $0.902-1.099$ \\
\hline Año 2007 & 0.878 & 0.013 & $0.792-0.973$ \\
\hline Día laboral & 1 & & \\
\hline Fin de semana & 0.505 & 0.000 & $0.452-0.563$ \\
\hline Festivo & 0.568 & 0.000 & $0.435-0.742$ \\
\hline Enero & 1 & & \\
\hline Febrero & 0.962 & 0.742 & $0.766-1.208$ \\
\hline Marzo & 0.987 & 0.909 & $0.792-1.229$ \\
\hline Abril & 1.073 & 0.531 & $0.859-1.342$ \\
\hline Mayo & 1.144 & 0.250 & $0.909-1.439$ \\
\hline Junio & 1.036 & 0.788 & $0.799-1.342$ \\
\hline Julio & 0.837 & 0.203 & $0.636-1.100$ \\
\hline Agosto & 0.864 & 0.286 & $0.660-1.130$ \\
\hline Septiembre & 1.066 & 0.613 & $0.831-1.367$ \\
\hline Octubre & 1.137 & 0.238 & $0.918-1.410$ \\
\hline Noviembre & 1.112 & 0.332 & $0.896-1.382$ \\
\hline Diciembre & 1.212 & 0.071 & $0.979-1.496$ \\
\hline $\mathrm{T}^{\circ}>25^{\circ} \mathrm{C}$ & 1.188 & 0.030 & $1.017-1.388$ \\
\hline Pres. Media/dia & 0.998 & 0.013 & $0.998-0.999$ \\
\hline
\end{tabular}

Como se observa en la tendencia el año 2007 es un año donde la siniestralidad laboral atendida ha sido un $14 \%$ menos que en el año 2005 , considerado como basal en el modelo.

Se observa que existe un Riesgo Relativo menor de atender un accidente laboral los fines de semana y los festivos.

En cuanto al comportamiento de los meses podemos ver que existe mas siniestralidad los de Mayo, Octubre y Diciembre y los que menos los de Julio y Agosto, aunque ninguno es significativo.

Los días con temperatura superior a $25^{\circ} \mathrm{C}$. el Riesgo Relativo de atender accidentes laborales en la puerta de urgencias es $19 \%$ mayor que si no se alcanzan esas temperaturas.

Respecto a la presión atmosférica media vemos que tiene un efecto protector, por cada milibar existe un cambio de $2 \%$ en el R. R. es decir los día con presión atmosférica alta suelen ser días con una menor asistencia de accidentes laborales.

Por último para representar gráficamente (gráfico 4) la comprobación del ajuste del modelo obtenemos el intervalo de confianza superior, al $95 \%$, del modelo estimado y lo representamos con los accidentes laborales observados. 


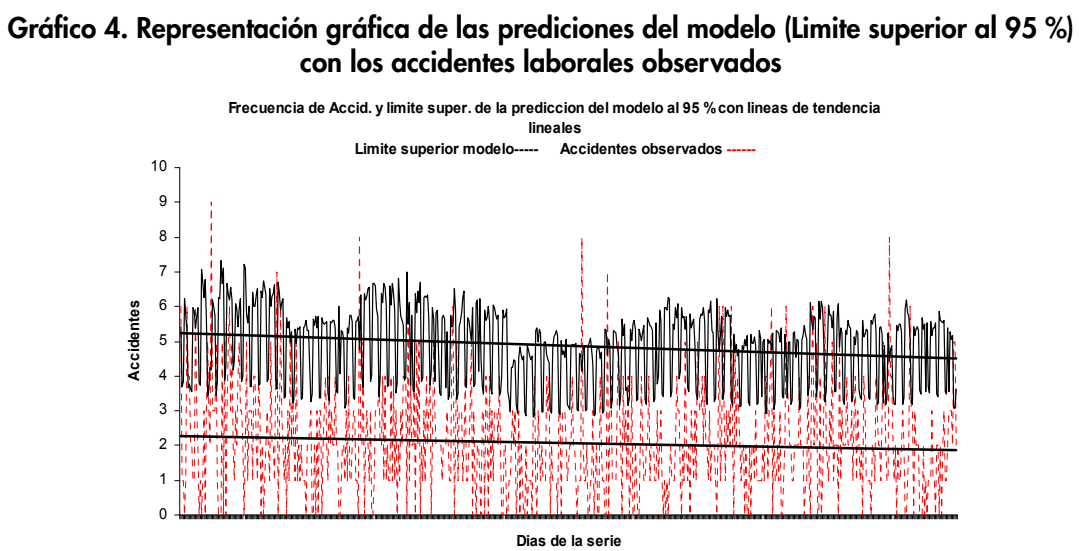

\section{DISCUSIÓN}

En primer lugar debemos tener presente que este tipo de análisis no son los adecuados para determinar la relación entre la exposición y el daño individual, es por tanto arriesgado extrapolar estos resultados a la siniestralidad en general, en este caso el A.T. Es lo que se denomina falacia ecológica o sesgo agregado.

Al tener presente que son contingencias laborales atendidas en Urgencias de un Hospital de Agudos es necesario considerar las características de estas, posiblemente, accidentes de tráfico, heridas y contusiones en muchas ocasiones graves, etc. Lamentable en las bases de datos obtenidas no figuraban los diagnósticos lo que consideramos una limitación importante.

Otra limitación del estudio es la ausencia de datos del sector de actividad en los que se producen, no pudiendo relacionar si estos accidentes ocurren con más prevalencia en algún sector en concreto, construcción, industria, servicios, etc. Sería importante observar si estos accidentes guardan relación o no, con actividades que se desarrollen a la intemperie.

También debemos resaltar las características geográficas del hospital, situado en el centro de Madrid y consecuentemente alejado de áreas de elevada actividad industrial, más proclive a la siniestralidad (Construcción, polígonos industriales, etc.), por lo que los resultados podrían no coincidir con otros centros hospitalarios o centros pertenecientes a Mutuas Laborales. Por otra parte, como ya comentamos al carecer de los diagnósticos, es posible que muchos de estos accidentes laborales atendidos en este hospital son debidos a accidentes de tráfico, bien in itinere o secundarios a la movilidad de los trabajadores en su actividad.

Es importante considerar que el marco temporal del estudio no es el deseado, en este tipo de estudio donde el número de años es escaso haciendo difícil la observación de la tendencia y de los ciclos. Esta limitación ha venido impuesta por los datos disponibles de contaminación y climatológicas cuyos registros, durante el trienio analizado, eran más homogéneos.

Las variables meteorológicas han sido recogidas de una estación en concreto (Getafe) y se han extrapolado a toda el área de Madrid, creemos que este aspecto no debe de influir excesivamente en los resultados.

Es lógico pensar que la frecuentación a puertas de urgencias hospitalarias en fines de semana y festivos es 2 veces menos que en el resto de los días de la semana, lo que quizás llame la atención es el comportamiento de la siniestralidad durante los meses, así el mes de diciembre que podríamos considerar significativo $(p=0.07)$, se obtiene un riesgo de accidentes del $67 \%$ más elevado que en el mes de Enero. No podemos explicar 
este motivo, pero posiblemente las fiestas navideñas puedan tener relación con este incremento.

Llama la atención que las variables de contaminación NO2 y PM10 son significativas en el análisis univariante. Estos son elementos importantes en la polución atmosférica y pudieran estar asociados con días de elevada actividad en general, tanto industrial como con días de elevado tráfico de vehículos y por tanto directamente relacionado con una mayor siniestralidad. No obstante en el modelo multivariante de Poisson definitivo no figuran, pensamos que posiblemente estén muy condicionadas por las variables climatológicas.

En las variables meteorológicas, es significativa, la presión atmosférica media del día, que se comporta como un efecto protector, aunque mínimo, por cada milibar disminuye el riesgo de atención urgente del accidente laboral en un $2 \%$. Posiblemente la explicación se deba a que los días climatológicamente más estables están más relacionados con la baja siniestralidad que los días en los que predominan otros meteoros (nieve, lluvia etc.) esta asociación también se ha observado en otros trabajos que han estudiado la mortalidad y variables meteorológicas ${ }^{5}$. Curiosamente las temperaturas elevadas (por encima de $25^{\circ} \mathrm{C}$ ) incrementan el número de accidentes laborales atendidos. Esta relación ya ha sido recogida por otros autores ${ }^{6,7,8}$.

Creemos que para profundizar en esta línea de trabajo sería conveniente proyectar un futuro estudio multicéntrico, con bases de datos mucho más extensas aportadas por las entidades encargadas fundamentalmente de la gestión de las contingencias laborales (Mutuas) con el fin de comprobar estos resultados y modelizar predicciones para un mejor conocimiento y prevención de los A. T.

\section{BIBLIOGRAFÍA}

1. Abanades Gracía JC, Cuadrat Prats JM, De Castro Muñoz de Lucas M, et al. El Cambio Climático en España. Estado de Situación. Madrid. Ministerio de Medio Ambiente; 2007. Disponible en: www.mma.es/secciones/ cambio _ climático

2. Plan Nacional de Adaptación al Cambio Climático. Oficina Española del cambio Climático.Disponible:www. mma.es/portal/secciones/cambio_climatico/areas_tematicas.

3. Ballester Diez f, Saez Zafra M, Alonso Fuster E, et al. El Proyecto EMECAM: Estudio Multicentrico Español sobre la relación entre la contaminación atmosférica y la mortalidad. Antecedentes, participantes, objetivos y metodología. Rev Esp Salud Pública 1999; 73: 165-175.

4. Saéz M, Pérez- Hoyos S, Tobías A, Taurina C, Barceló MA, Ballester F. Métodos de series temporales en los estudios epidemiológicos sobre contaminación atmosférica. Rev Esp Salud Pública 1999; 73: 133-143.

5. Gonzalez S, et al. "Relationship between atmospheric pressure and mortality in the Madrid Autonomous Region: a time-serie study. International journal of Biometerology. 2002; 45: 34 -40.

6. Morabito M, Cecchi L, Crisci A, et al. Relationship between work-related accidents and hot weather conditions in Tuscany (Central Italy). Industrial Health 2006; 44: 458 - 464.

7. Tanaka M. Heat Stress Standard for Work Enviorenments in Japan. Industrial Health 2007; 47: 85 -90.

8. Schwartz J, Samet JM, Patz JA. Hospital admissions for heart disease : the effects of temoperature and humidity. Epidemiology 2004; 15:755 - 61.

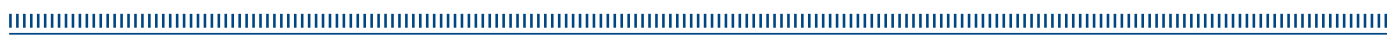

